\title{
Vitamin D deficiency and Bone health in patients with Learning Disability
}

Nataliya Zuyeva, Abbi Lulsegged, Robert Winterhalder

\section{Introduction}

There is limited data on vitamin $D$ status in patients with learning disabilities (LD). As a group they possess multiple risk factors for vitamin $D$ deficiency including limited access to sunlight, problems with mobility and anticonvulsant use.

The purpose of this study was to evaluate vitamin $D$ status in adults with LD living in nursing homes and to compare serum vitamin D ( S-25-OHD) level in people with different degree of LD to establish the dose of vitamin D3 needed to correct the vitamin $\mathrm{D}$ deficiency with the aim of correcting above a minimum target level of $80 \mathrm{nmol} / \mathrm{L}$ or $32 \mathrm{mcg} / \mathrm{L}$.

\section{Materials and Methods}

The study included 105 adults (66 males and 39 females), 19-73 years of age $(39 \pm 1.6)$ with learning disability living in a nursing homes in London. Patients were divided according to the severity of learning disability (profound, severe, moderate, mild). Almost all of them had epileptic syndromes of different etiology and 103 patients were on different types of antiepileptic medications.

Clinical data on the etiology and severity of LD, other illnesses, medications, anthropometry were collected from medical records. The participants were receiving different doses of vitamin D3 (400 - $3000 \mathrm{IU})$ daily per orally for 6 months.

Blood samples were obtained at baseline and at 6 months for parameters of calcium homeostasis, including serum

concentrations of 25-hydroxyvitamin D (S-25-OHD) . Data was available on vitamin $D$ and calcium levels.

All data are expressed as the mean \pm SE

\section{$\underline{\text { Results }}$}

Both the baseline and post-treatment concentration of vitamin $D$ did not differ between male and female patients (Figure 1). There was a very weak negative correlation between baseline 25vitamin $D$ levels and the degree of learning disability $(r=-0.14)$. There was no significant difference between the 4 groups before treatment, however there was a clear trend of higher vitamin $D$ levels with a significantly higher post treatment vitamin $D$ level in the profound learning disability group compared to those with mild deficiency (Figure 2).

The average dose of vitamin $D 3$ needed to increase the vitamin $D$ level above $80 \mathrm{nmol} / \mathrm{L}$ was 1945 units daily.

\section{Conclusion}

Our data shows that in a group of patients with significant risk factors of vitamin $D$ deficiency, the average dose of vitamin $D$ needed to correct deficiency wason average a modest dose given the need for anticonvulsants. Indeed the response to treatment was greatest in the group who had the most profound disability.
B

Figure 1. Serum vitamin D3 concentration in male and female patients with learning disabilities before $(A)$ and after $(B)$ treatment with vitamin D3
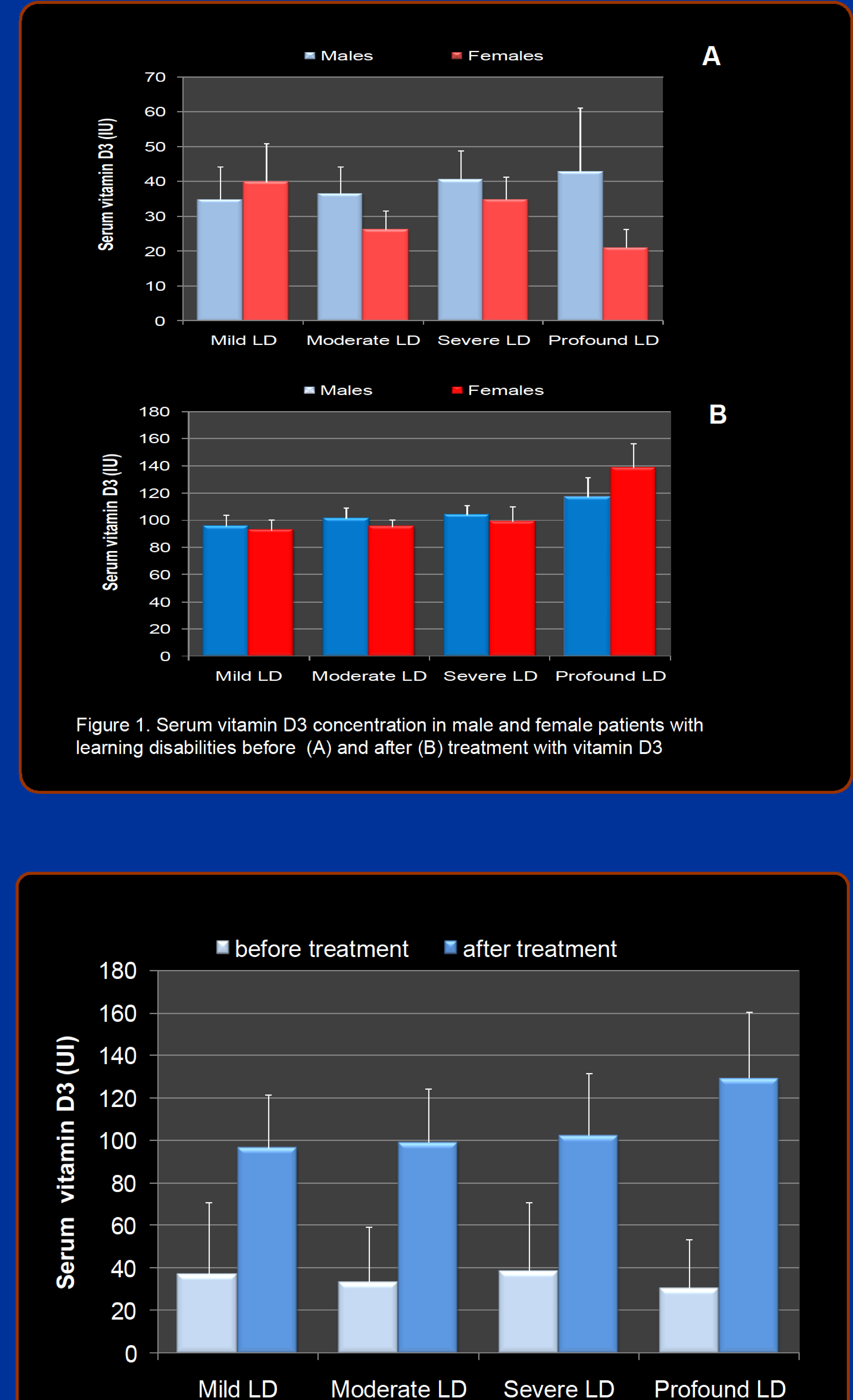

Figure 2. Serum vitamin D3 concentration in patients with the different severity of learning disability before and after treatment with vitamin D3 